\title{
Signal processing for T-ray bio-sensor systems
}

\author{
Bradley Ferguson* and Derek Abbott \\ Centre for Biomedical Engineering and \\ Department of Electrical and Electronic Engineering, \\ Adelaide University, SA 5005, Australia
}

\begin{abstract}
Pulsed terahertz (T-ray) imaging systems represent an extremely promising method of obtaining sub-millimetre spectroscopic measurements for a wide range of applications. This paper investigates a number of techniques for optimally processing terahertz data. Specifically we consider wavelet de-noising and Wiener deconvolution algorithms.

A goal of this research is the design and implementation of a high speed, compact and portable T-ray imaging system. This system will draw heavily on MOEMS technology. A significant challenge in the development of such a system is the development of efficient software algorithms to perform signal recognition and imaging operations in real time. This paper takes the example application of a smart bio-sensor for surface tumours and investigates the signal processing techniques amenable to the tasks of efficiently de-convolving the system response, de-noising and extracting the salient features from the terahertz response waveform.
\end{abstract}

Keywords: terahertz, T-ray imaging, wavelet de-noising, Wiener deconvolution

\section{INTRODUCTION}

Signal processing for pulsed terahertz systems is a relatively unexplored field that promises to make a significant contribution to the speed and accuracy of modern T-ray systems. This paper explores a number of different signal processing methods applied to the data recorded from an all optical terahertz pulse imaging system. The different approaches are compared both theoretically and practically and their relative benefits discussed.

Terahertz radiation has a number of unique characteristics, which give rise to a large number of potential applications in very diverse fields. Non-polar, dry substances, such as cardboard and plastics, are transparent to terahertz radiation; T-rays can therefore be used for integrated circuit and packaging inspection and quality control. ${ }^{1}$ Water and other polar liquids absorb strongly, thus opening applications in moisture analysis. ${ }^{2}$ Many molecules have characteristic rotational spectra in the terahertz regime and T-ray systems have been used for gas sensing and analysis. $^{3}$ Terahertz radiation also has significant advantages for imaging applications, especially biomedical imaging. Historically biomedical imaging has relied on visible imaging and microwave (RF) imaging. Visible imaging is limited by Rayleigh scattering which is inversely proportional to the fourth power of the wavelength. This scattering causes attenuation and blurring of the image. ${ }^{4}$ Microwave imaging operates at much longer wavelengths but is limited to resolutions of the order of $1 \mathrm{~cm}$. Terahertz radiation represents an ideal trade-off. Its wavelength is long enough to prevent drastic Rayleigh scattering yet short enough to retain fine resolution. As an added advantage terahertz radiation is non-ionising and thus represents a totally non-invasive diagnostic technique. ${ }^{5}$

Modern terahertz systems suffer limitations in terms of resolution, accuracy and speed. Much current research is focussed on overcoming these obstacles by improving the system hardware. This paper describes research aiming to address these issues from a different perspective - through the use of digital signal processing.

There are several sources of noise in terahertz systems, due to both systematic and random errors. One method to reduce errors due to noise is to average subsequent measurements for the same sample, however this drastically increases the time required to perform the measurement. Signal processing potentially offers an improved solution to the noise problem.

Wavelets are a critical interest in this research as they possess a range of extremely attractive properties for this application. This paper includes an evaluation of the quality of wavelet de-noising of terahertz waveforms and a comparison of the different wavelet bases with respect to the bio-sensor application.

* Correspondence: Email: bradleyf@eleceng.adelaide.edu.au, dabbott@eleceng.adelaide.edu.au; Telephone: +61 88303 6296; Fax: +61 883034360

Smart Electronics and MEMS II, Derek Abbott, Vijay K. Varadan, Karl F. Boehringer, Editors, Proceedings of SPIE Vol. 4236 (2001) (C) 2001 SPIE · 0277-786X/01/\$15.00 
Another significant source of errors and ambiguity in terahertz systems is the system hardware itself. The signal obtained for a sample is a result of the far-infrared properties of the sample (which are of critical interest) and the properties and non-idealities of the system itself, these include electrical and optical reflections for system components and numerous other effects. ${ }^{6}$ In order to accurately analyse the results it is vital that the sample signal characteristics be isolated from the system characteristics. This is generally performed by the process of deconvolution. The standard deconvolution process is extremely sensitive to noise and can result in considerable errors when noise is present. Improved methods of deconvolution which can optimally deconvolve a noisy signal are a major subject of this paper.

This paper describes the software algorithms under development for a T-ray imaging system. Section 2 provides an overview of the hardware, the sources and characteristics of noise in the system and the current state of the art in signal processing for such systems. Section 4 defines the signal processing problem from a mathematical view point to formalise the setting for the following discussion. Section 5 discusses the application of wavelet de-noising to the problem and addresses the question of choosing an ideal wavelet family to maximise the performance of the de-noising algorithm. Section 6 considers the problem of deconvolving the T-ray data. It presents the formulation of an ideal filter (in the sense of the mean square error) to deconvolve the sample response in the presence of noise. The deconvolution problem is addressed in the frequency domain, by the means of the Wiener filter. The penultimate section, Section 7 gives a visual comparison of the effects of the processing algorithms, and finally Section 8 summarises this work and presents an overview of the future work planned in this developing field.

\section{MODERN T-RAY SYSTEMS}

The technique of terahertz pulse imaging has been used for over 10 years $^{7}$ in a wide range of applications, from measuring optical constants of semi-conductors, ${ }^{8}$ gas sensing ${ }^{9}$ to moisture analysis ${ }^{2}$ and beyond. ${ }^{5}$ It is a powerful technique with enormous potential. Its potential is a result of several characteristics that distinguish it from competing technologies. These include the unique properties displayed by many substances in the terahertz frequency range, the broadband nature of the emitted radiation and the coherent detection technique which allows amplitude and phase information to be obtained.

Terahertz systems have been proposed for a number of biomedical applications including the detection of tooth cavities, ${ }^{10}$ and burn severity diagnosis. ${ }^{5}$ One of the most potentially significant biomedical applications is in the detection of skin cancers such as malignant melanoma and basal cell carcinoma. The incidence of these cancers continue to escalate and in the advanced stages there is no curative therapy available, ${ }^{11}$ early detection is therefore of prime importance. Currently most dermatologists rely on a visual assessment of the patient for diagnosis. This diagnosis is not straight-forward and results in a large number of cases being treated inappropriately or inadequately. Hence there is considerable interest in the development of a non-invasive technique to improve clinician's diagnostic accuracy. Recently researchers have investigated the use of white light reflectance spectroscopy for this purpose. ${ }^{12}$ They have found significant differences in the spectra for malignant and benign lesions which would allow automated diagnosis. These studies have focussed on calculating metrics such as the tissue hemoglobin concentration, water concentration and hemoglobin oxygen saturation. ${ }^{13}$ A similar system based on the principle of terahertz time-domain spectroscopy has the potential to perform the same task with the added advantages of reduced Rayleigh scattering and finer frequency resolution. We present experimental results analysing biological samples with terahertz radiation. Ideally this system will be implemented using MOEMs technology to allow a portable system to be developed and research is being conducted in this field. ${ }^{14}$

It is only recently that attention has been given to improving the performance of T-ray systems through the use of signal processing techniques. The major goal of most experiments involves the determination of the complex, frequency-dependent refractive index of the substance under test. In the vast majority of cases this has been calculated using the following simple procedure. The time-domain terahertz pulse is measured using the system shown in Figure 1 without a sample in place. This signal is referred to as the system response. The sample is then added to the system and the terahertz pulse measured again. The Fourier transforms of these two signals are found and then the ratio of the transforms yields the complex transmission coefficient of the sample as a function of frequency. ${ }^{15,16}$ Taking the ratio in the frequency domain performs the deconvolution of the signal and serves to isolate the sample dependent characteristics. Subsequent processing is then application dependent. For dielectric and semiconductor characterisation the refractive index can be derived from the transmission coefficients, ${ }^{17}$ whereas for gas sensing applications the transmission frequency response is analysed to identify absorption spectra corresponding to specific 
molecular resonances. Signal processing techniques such as linear predictive coding (LPC) have been employed to improve the sensitivity in gas sensing and chemical recognition applications. ${ }^{6,9}$

One of the significant problems posed by the above processing is the inherent noisiness of the deconvolution process. The simple ratio deconvolution amplifies any high frequency noise in the signal. ${ }^{18}$ One method of countering this problem is through the application of a pre-processing filter. Wavelet de-noising filters have been suggested due to their pulse-like nature. ${ }^{6,19}$ Another approach to this problem is the Wiener filter ${ }^{6}$ which performs system deconvolution and also performs optimal noise cancellation in the mean-square error sense. Both of these ideas are analysed in this paper.

Figure 1 provides a simplified schematic of the hardware used for terahertz pulse imaging. This system has been described many times in the literature, ${ }^{7,20,21}$ however, a brief overview is warranted here to identify the major components and the potential sources of noise.

The basic system components consist of a femtosecond laser, a variable delay line, an optically gated $\mathrm{THz}$ transmitter and detector and an analog to digital converter. A lock-in amplifier (LIA) may be used to digitise the signal. The LIA can provide a large advantage in signal to noise ratio of up to 100 times at the expense of acquisition speed. ${ }^{21}$ Figure 2 shows the effect of the LIA time constant on the measurement noise.

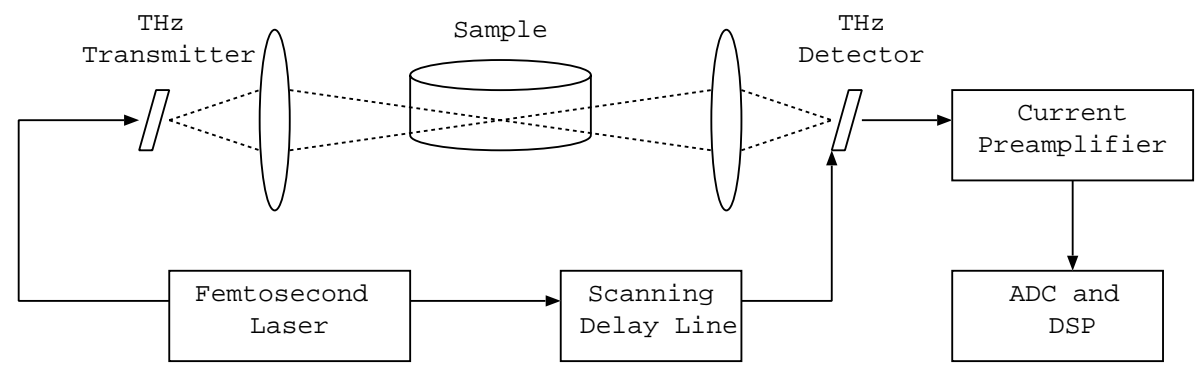

Figure 1. Simplified hardware schematic used for terahertz time-domain spectroscopy.

There are a large number of noise sources in terahertz systems. The relative intensity of these sources varies depending upon the particular setup and sample under test, but it is generally found that the emitter noise dominates all other noise contributions. This noise is a result of random intensity fluctuations in the ultrafast laser and has been quite extensively studied. ${ }^{22,23}$ Other major sources of random error are Johnson and shot noise in the THz detector $^{24}$ as well as thermal background radiation in the $\mathrm{THz}$ regime. The coherent nature of T-ray systems allow them to achieve impressive performance despite very high relative noise magnitudes. The noise is incoherent and adds randomly for successive gating pulses while the signal is coherent and scales linearly with the number of gating pulses. ${ }^{7}$

In addition to these random errors there are several potential systematic errors in the system. These include parasitic reflections of the $\mathrm{THz}$ beam from system components, phase errors due to delay line misalignment and absorption and dispersion of water vapour. ${ }^{3}$

To investigate the relative benefits of different signal processing methods a standard set of real T-ray data was used. It consists of a $100 \times 100$ pixel image of an oak leaf with an insect sitting on the side of the leaf as shown in Figure 4. The image has a spatial resolution of approximately $1 \mathrm{~mm}$. For each pixel the time response of the terahertz pulse was recorded over $12 \mathrm{ps}\left(10^{-12} \mathrm{~s}\right)$ at an effective sample rate of 25 terasamples/second. Examples of these responses for each of the three distinct media (leaf, insect and free air) are shown in Figure 5.

\section{DEFINITIONS AND NOTATION}

The signals considered here, $f(k), k \in 0 . . N-1$, are real-valued, finite, discrete-time functions defined on $\mathbb{R}$.

The inner product of two sequences $f$ and $g$ is written

$$
<f, g>=\sum_{k=0}^{N-1} f(k) g(k) .
$$



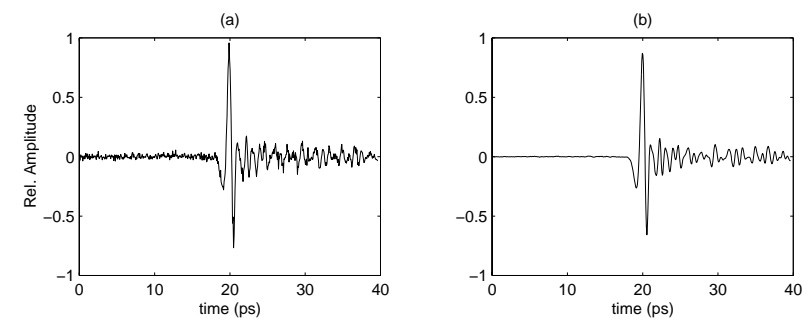

Figure 2. Terahertz responses of an Australian $\$ 100$ note obtained with different LIA time constants. (a) was measured with a time constant of $100 \mathrm{~ms}$. (b) was measured with a time constant of $1 \mathrm{~ms}$. The pixel shown corresponds to the ink of the 0 digit in the upper right corner of the note.

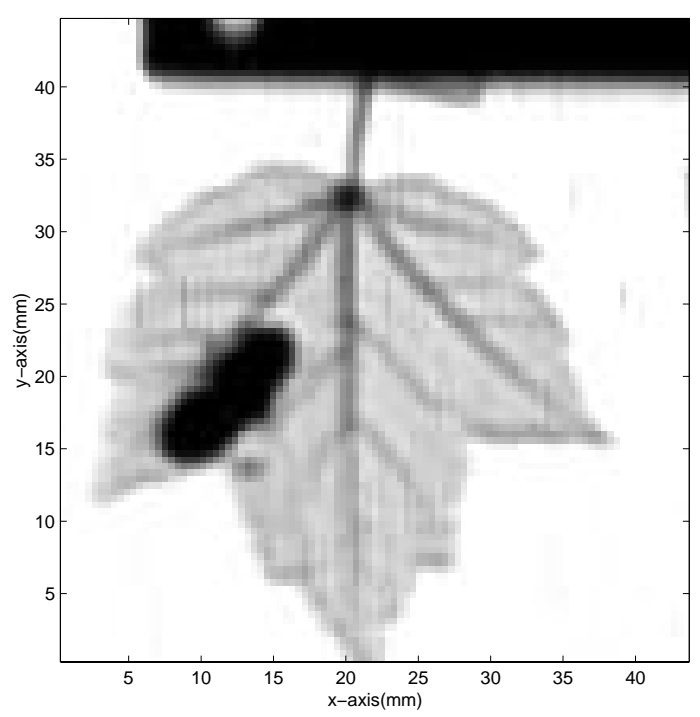

Figure 4. Raw image of an insect on an oak leaf obtained by terahertz time-domain spectroscopy. The image is produced by taking the Fourier transform of the response for each pixel and plotting the maximum of the Fourier coefficients.

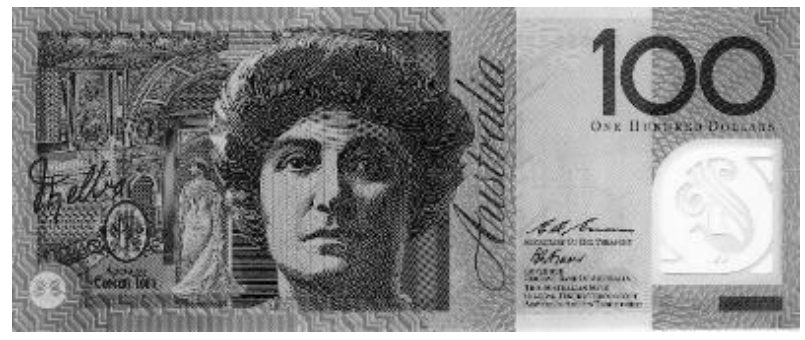

Figure 3. Standard optical image of an Australian $\$ 100$ note.
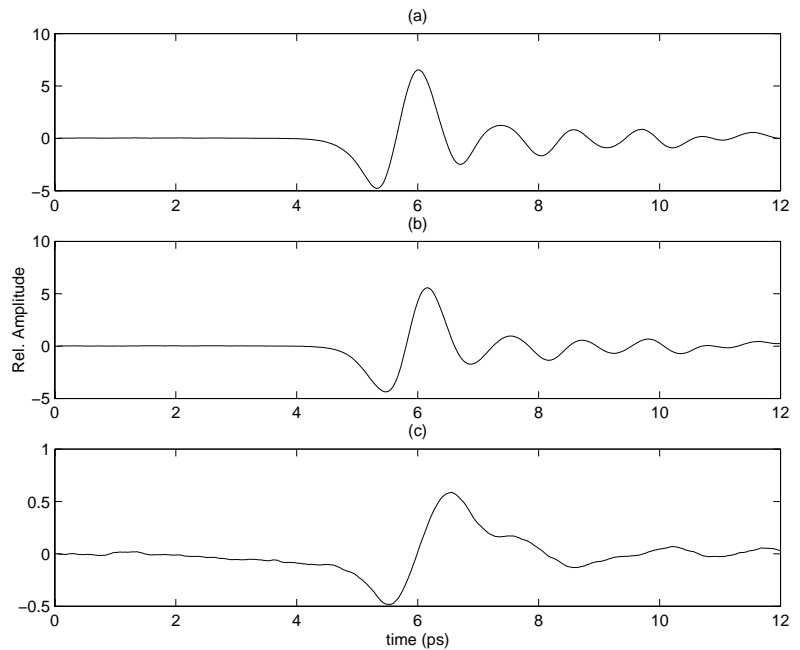

Figure 5. Sampled terahertz pulses after transmission through various substances: (a) air, (b) oak leaf, and (c) an insect. 
The dyadic, discrete wavelet transform of a sequence $f$ with respect to the mother wavelet $\psi$ is given by

$$
\begin{aligned}
d_{i}=W_{\psi} f(m, n) & =\frac{1}{\sqrt{2^{m}}} \sum_{k=0}^{N-1} f(k) \psi\left(\frac{k-n 2^{m}}{2^{m}}\right), \\
& =\left\langle f, \psi_{m, n}\right\rangle .
\end{aligned}
$$

The Fourier transform of a sequence $f(k)$ is denoted by $F(\omega)$ and is given by

$$
F(\omega)=\sum_{k=0}^{N-1} f(k) e^{-i \omega k}
$$

The discrete convolution between two sequences $f$ and $g$ is the sequence $f * g$ given by

$$
(f * g)(l)=\sum_{k=0}^{N-1} f(k) g(l-k) .
$$

\section{PROBLEM DEFINITION}

We consider the problem of determining the complex, frequency-dependent transmission coefficients for a given sample, whether that be a gas, a semiconductor, a leaf or human tissue. Figure 6 illustrates the system and signals we are considering.

Let $x(k)$ be the measured terahertz response for free air pixel, $\mathrm{i}$,

$$
x(k)=f(k)+n_{i}(k), \quad k=0, \ldots, N-1,
$$

where $f(k)$ is the input signal generated by the terahertz emitter and $n_{i}(k)$ is Gaussian white noise.

Similarly let $y(k)$ be the terahertz response obtained for a pixel, $\mathbf{j}$, containing the sample under test

$$
y(k)=g(k)+n_{j}(k), \quad k=0, \ldots, N-1,
$$

where $g(k)$ the output of the unknown system when $f(k)$ is applied and $n_{j}(k)$ is Gaussian white noise. Then we model the response of the sample as a linear, time-invariant system:

$$
\begin{aligned}
g(k) & =f(k) * h(k), \\
y(k) & =f(k) * h(k)+n_{j}(k), \\
& =\left(x(k)-n_{i}(k)\right) * h(k)+n_{j}(k),
\end{aligned}
$$

where $h(k)$ is the impulse response of the sample under test.

The goal then is to design an algorithm to optimally determine $h(k)$ and $H(\omega)$ of the sample in the mean square error sense, given the noisy measured signals $x(k)$ and $y(k) .^{*}$

\section{WAVELET DE-NOISING}

\subsection{General Wavelet Theory}

If $f(k)$ and $g(k)$ can be estimated from the noisy observations the problem can be simply solved from Equation 7 . Wavelet de-noising represents a promising method in this regard. The theory of the wavelet transform has existed for many years in a number of forms. In the late 1980's Stéphane Mallat unified the various theories and coined the term: 'the Wavelet Representation'. ${ }^{25}$ Since then wavelets have found application in a wide range of fields as a result of their attractive and efficient properties.

\footnotetext{
${ }^{*}$ Note that the above analysis is a simplification of the problem to aid tractability. In practice the measured sample response is determined by the non-linear responses of the sample, the $\mathrm{THz}$ emitter and the detector.
} 

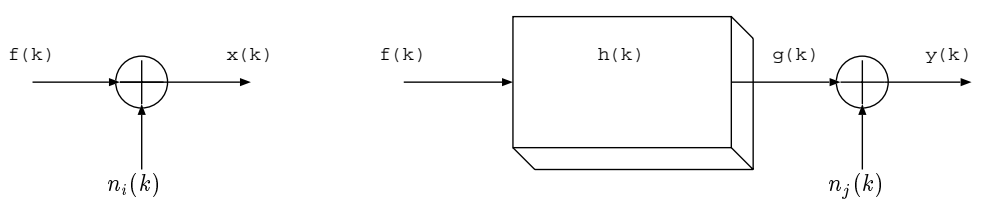

Figure 6. Block diagram illustrating the elements necessary to define the problem. (a) shows the cases where the sample is not present, (b) shows the case when the sample is included.

Wavelet methods are extremely efficient and computationally inexpensive. The fast Discrete Wavelet Transform (DWT) allows wavelet coefficients to be calculated with a computational complexity of $\mathbb{O}(\mathrm{N})$.

Wavelets have been shown to be particularly useful in the analysis of non-stationary signals and in image processing. This is because the wavelets, which form the basis functions for the transform, are localised in both time and frequency. This is in contrast to the Fourier transform, which uses infinite sinusoids as the basis functions.

The time-frequency localisation of the wavelet basis functions make the wavelet transform a more efficient representation of pulsed functions such as terahertz pulses. This allows for more effective de-noising, compression and statistical estimation than Fourier analysis. In fact, it has been shown that wavelet bases are optimal for representing functions containing singularities. ${ }^{26}$ The goal of the wavelet transform in our context is to allow the measured signal to be separated into coherent structure and incoherent noise.

Wavelet shrinkage de-noising is widely used for this purpose. Donoho ${ }^{27}$ defined the process of soft thresholding in the wavelet domain and proved that it was optimal in the mean square error sense. Soft thresholding is performed via the following procedure:

1. Determine the wavelet coefficients, $d_{i}$, by taking the wavelet transform (Equation 2).

2. Calculate the threshold value, $T$,

$$
T=\sigma \sqrt{2 \log _{e} N}
$$

where $\sigma$ is the noise level and $N$ is the number of samples,

3. Threshold the wavelet coefficients by moving them all towards zero by the threshold amount,

$$
d_{i}=\left\{\begin{array}{lll}
d_{i}-T & \text { if } & d_{i} \geq T \\
d_{i}+T & \text { if } & d_{i} \leq-T \\
0 & \text { if } & \left|d_{i}\right|<T
\end{array}\right.
$$

4. Perform the inverse wavelet transform to recover the time domain signal.

More advanced algorithms have been proposed for de-noising and signal analysis. These include wavelet packets, ${ }^{28}$ matching pursuits ${ }^{29}$ and many others. However, this study focuses solely on the simple de-noising procedure outlined above.

\subsection{Choosing a Wavelet Family}

A large number of wavelet bases have been developed for different applications. Some examples of these wavelets are shown in Figure 7. Figure 8 shows the wavelet coefficients for the leaf T-ray response when decomposed using the example wavelet bases. These wavelets each exhibit different properties and the following discussion addresses the question of determining the ideal mother wavelet for representing and de-noising the T-ray data shown in Figure 5.

The goal of wavelet de-noising is to approximate the noiseless function with as few non-zero wavelet coefficients as possible. The wavelet family $\psi$ should therefore be chosen to produce a maximum number of wavelet coefficients that are close to zero. The main properties of the wavelet that will affect this are its regularity, the number of vanishing moments and the compactness of its support. ${ }^{30}$ 

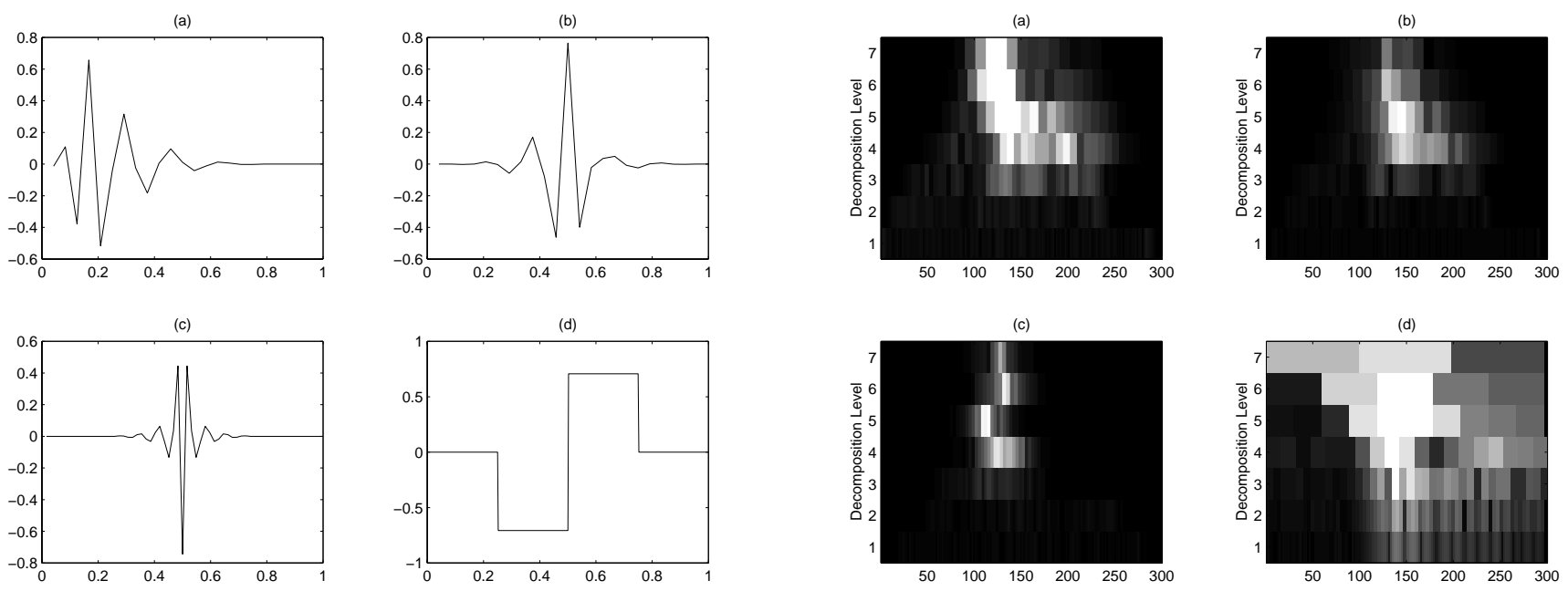

Figure 7. Examples of different wavelet basis functions, $\psi$ : (a) Daubechies (Order 12), (b) Symlet (Order 12), (c) Meyer, and (d) Haar (Daubechies order 1).

Figure 8. Example of wavelet coefficients for a Tray response using different wavelet basis functions: (a) Daubechies order 12, (b) Symlet order 12, (c) Meyer, and (d) Haar (Daubechies order 1).

A wavelet $\psi$ has $p$ vanishing moments if

$$
\int_{-\infty}^{+\infty} t^{k} \psi(t) d t=0 \quad \text { for } 0 \leq k<p .
$$

Thus $\psi$ is orthogonal to any polynomial of degree $p-1$. If $f$ is regular and can be approximated over a small interval by a Taylor polynomial of degree $k$ and if $k<p$, then the wavelet is orthogonal to this polynomial and the wavelet coefficients will be small for fine scales (high-resolution), thus for smooth functions a wavelet with a higher number of vanishing moments will represent the function with fewer large coefficients.

The size of support of a wavelet $\psi$ refers to the range over which $\psi$ has non-zero values, that is, it is a measure of the temporal localisation of the wavelet. The wider the support of the wavelet the more large amplitude coefficients are generated by peaks in the input signal $f$, this is a particular problem if the signal has many isolated singularities.

To reduce the number of large amplitude coefficients we must reduce the support size and increase the number of vanishing moments of $\psi$. However, it can be shown ${ }^{30}$ that if $\psi$ has $p$ vanishing moments then its support is at least $2 p-1$. Therefore some trade off must be made. Daubechies wavelets are optimal in this regard as they offer the minimum support for a given number of vanishing moments. ${ }^{31}$

The regularity of $\psi$ has a limited effect on thresholding efficiency. Its main importance is in the field of image processing where irregular wavelets can result in obvious discontinuous artifacts in the processed image. Other properties that distinguish between wavelets are their symmetry and orthogonality.

We limit this investigation to orthogonal, dyadic wavelet transforms due to the very fast algorithms available for their calculation, however many applications such as speech processing require non-dyadic wavelet processing to accurately represent subtle changes in scale and delay. ${ }^{32}$ Non-dyadic wavelet processing thus represents an open area for future research.

\subsection{Experimental Determination of the Optimal Wavelet}

Experiments were performed to determine which wavelet family performed best in de-noising noisy T-ray data. To do this white, Gaussian noise was added to the measured terahertz responses such that the SNR was $3 \mathrm{~dB}$, the noisy sequence was then de-noised using Daubechies, Meyer, Symlet and Coiflet wavelets of varying order. The order of the wavelet, $\mathrm{N}$, is of particular concern to this discussion as it determines both the number of vanishing moments and the support length for the wavelet. Table 1 shows the properties of the wavelets considered here. More details on the wavelet properties can be found in the literature. ${ }^{30,31}$ 
Table 1. Comparison of the major properties of the wavelets considered in this study.

\begin{tabular}{|c|c|c|c|c|}
\hline \multirow{2}{*}{ Characteristic } & \multicolumn{3}{|c|}{ Wavelet } \\
\cline { 2 - 5 } & Meyer & Daubechies & Coiflet & Symlet \\
\hline \hline Regularity & infinite & low, increases with N & increases with N & increases with N \\
Support Length & infinite & $2 \mathrm{~N}-1$ & $6 \mathrm{~N}-1$ & $2 \mathrm{~N}-1$ \\
Symmetric & yes & no & nearly & nearly \\
\# of Vanishing Moments & 0 & $\mathrm{~N}$ & $2 \mathrm{~N}$ & $\mathrm{~N}$ \\
Orthogonal & yes & yes & yes & yes \\
\hline \hline
\end{tabular}

The quality of the de-noising process was measured based on the resultant signal to noise ratio (SNR) given by

$$
\operatorname{SNR}(\mathrm{dB})=10 \log \left(\frac{\sum_{k=0}^{N-1} a(k)^{2}}{\sum_{k=0}^{N-1}[a(k)-b(k)]^{2}}\right),
$$

where $a(k)$ was the original terahertz signal and $b(k)$ was the final signal after noise has been added and wavelet de-noising performed.

This process was repeated over 100 times to average the results. This procedure was carried out for each of the characteristic T-ray responses, the free air, leaf and insect responses. Table 2 provides a summary of the results.
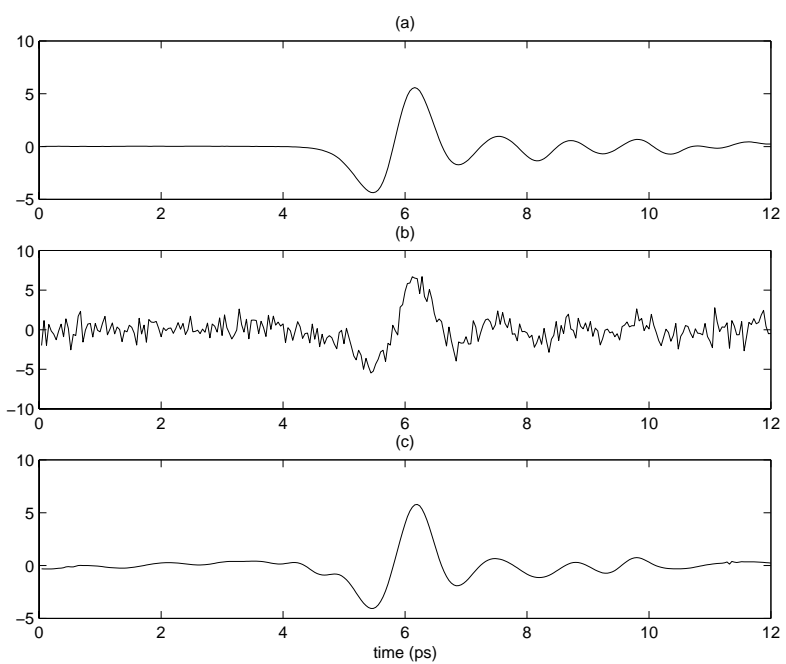

Figure 9. Results of wavelet de-noising the T-ray response for a leaf. (a) shows the original response before noise was added, (b) shows the response in (a) with noise added such that the SNR was $3 \mathrm{~dB}$, (c) shows the response in (b) after wavelet de-noising was performed using the Coiflet order 4 wavelet.

The results indicated that the wavelet de-noising procedure was very successful in de-noising the terahertz pulses, providing an improvement in SNR ranging from $5 \mathrm{~dB}$ up to $10 \mathrm{~dB}$. A typical result of the de-noising process is shown for the leaf terahertz response in Figure 9. All of the wavelets tested provided similar results however by averaging the results over each wavelet family the families could be ranked in order of decreasing quality to yield: Coiflet, Symlet, Daubechies and Meyer. For each wavelet family the variation of de-noised SNR with order was analysed. It was found that for each family there was an ideal order, $N^{*}$ for which the de-noised SNR was maximised. For orders $N<N^{*}$ the SNR dropped off rapidly and for higher orders $N>N^{*}$ the de-noised SNR dropped off more gradually in a complex fashion approximating an exponential decay overlaid with an oscillatory function. An example of this 
Table 2. Experimentally determined SNR for T-ray pulses de-noised with different wavelets of varying order. White noise was added to the original signals such that the signal to noise ratio was $3 \mathrm{~dB}$ before the denoising process was applied.

\begin{tabular}{|c|c||c|c|c|}
\hline \multirow{2}{*}{$\begin{array}{l}\text { Wavelet } \\
\text { Family }\end{array}$} & \multicolumn{1}{|c||}{ Order } & \multicolumn{3}{c|}{ De-Noised SNR (dB) } \\
\cline { 3 - 5 } & & Free Air & Leaf & Insect \\
\hline \hline Daubechies & 1 & 8.4 & 7.9 & 10.1 \\
& 5 & 11.2 & 11.9 & 13.3 \\
& 10 & 11.6 & 11.0 & 12.1 \\
& 15 & 10.8 & 10.5 & 10.8 \\
& 20 & 10.4 & 10.4 & 12.1 \\
& 25 & 9.5 & 8.4 & 11.3 \\
Coiflet & 1 & 10.6 & 10.5 & 11.5 \\
& 2 & 11.7 & 11.4 & 11.4 \\
& 3 & 12.0 & 11.7 & 11.4 \\
& 4 & 12.5 & 12.1 & 13.3 \\
& 5 & 12.0 & 12.1 & 12.4 \\
& 1 & 9.1 & 8.3 & 10.0 \\
& 5 & 10.9 & 11.7 & 13.2 \\
& 10 & 11.9 & 12.0 & 11.7 \\
& 15 & 10.6 & 11.4 & 12.1 \\
& 20 & 10.7 & 11.4 & 12.2 \\
& 25 & 10.6 & 10.5 & 13.7 \\
Meyer & undef & 11.4 & 11.8 & 13.9 \\
\hline \hline
\end{tabular}

Table 3. Experimentally determined ideal order for each wavelet family.

\begin{tabular}{|c||c|c|c|}
\hline \multicolumn{1}{|c||}{ Wavelet } & \multicolumn{3}{c|}{ Ideal Order } \\
\cline { 2 - 4 } Family & Free Air & Leaf & Insect \\
\hline \hline Daubechies & 7 & 8 & 8 \\
Coiflet & 4 & 5 & 4 \\
Symlet & 6 & 7 & 11 \\
\hline \hline
\end{tabular}

response is shown in Figure 10 for the Daubechies family. The ideal order for each wavelet was also found and is shown in Table 3.

\section{DECONVOLUTION}

This section considers the problem of estimating the impulse and frequency response of the sample under test from the measured data, as formulated in Section 4.

\subsection{The Wiener Filter}

The Wiener filter is the classic filter used for noise reduction. ${ }^{18}$ Given a signal $s(k)$ corrupted by noise $n(k)$, we desire to filter the resulting signal to yield $y(k)$ as close as possible to $s(k)$. If we assume that the signal and noise are ergodic, random variables of known power spectral densities (PSD), and further that the noise is uncorrelated with the signal, the optimal filter in the sense of mean, square error is given by

$$
H_{o}(\omega)=\frac{P_{s}(\omega)}{P_{s}(\omega)+P_{n}(\omega)},
$$

where $H_{o}(\omega)$ is the frequency response of the filter, and $P_{s}(\omega)$ and $P_{n}(\omega)$ are the PSDs of the signal and the noise respectively. These are estimated using the direct periodogram $\operatorname{method}^{33}$ whereby

$$
P_{s}(\omega)=\frac{1}{N}|X(\omega)|^{2}, \quad X(\omega)=\sum_{n=0}^{N-1} x(n) e^{-j \omega n},
$$

where $N$ is the number of samples, $x(n)$ is the input sequence.

The process of deconvolution involves dividing by the system frequency response. If this process is followed by a Wiener filter it is referred to as Wiener deconvolution. Figure 11 illustrates this process. 


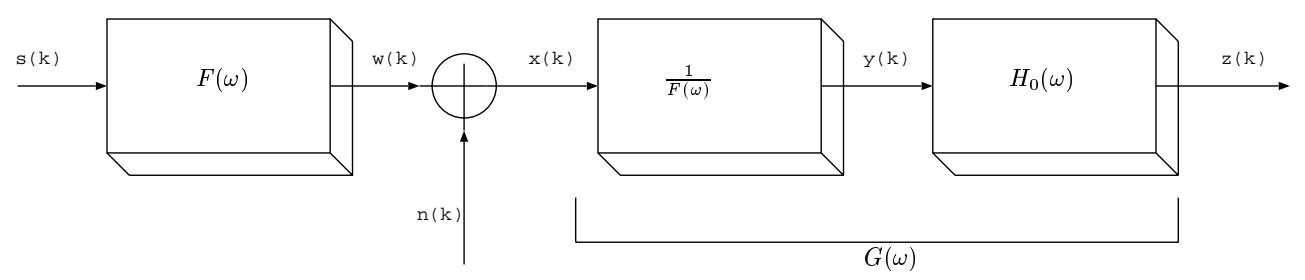

Figure 11. Block diagram illustrating the process of Wiener deconvolution. $G(\omega)$ represents the Wiener deconvolution filter. It consists of a deconvolution filter, followed by a Wiener filter.

The transfer function of the deconvolution filter $G(\omega)$ is given by

$$
G(\omega)=\frac{H_{o}(\omega)}{F(\omega)}=\frac{F^{*}(\omega) P_{s}(\omega)}{|F(\omega)|^{2} P_{s}(\omega)+P_{n}(\omega)} .
$$

\subsection{Experimental Deconvolution}

The Wiener filter described above was applied to terahertz data obtained by imaging a 0.6 mm thick slice of Spanish ham ('Jamon Serrano'). The data was obtained using the setup shown in Figure 1 using a 'Hurricane' laser from Spectral Physics, with a pulse repetition rate of $1 \mathrm{kHz}$, a pulse duration of $130 \mathrm{fs}$, a pump power of $50 \mathrm{~mW}$ and a wavelength of $800 \mathrm{~nm}$. Two pixels were considered, one was an area with a high concentration of fat, the other was a meaty area with a low fat concentration, the frequency spectra for these two samples are shown in Figure 12. Wiener deconvolution was applied to each of these samples, for this purpose the Fourier transform of the free air T-ray pulse $x(k)$ was used as $F(\omega)$, the sample T-ray response $y(k)$ was used to determine $P_{s}(\omega)$ and Gaussian white noise was added to both sequences such that the SNR of each sequence was $3 \mathrm{~dB}$. This was done to illustrate the performance of the filter in the presence of a large amount of noise.
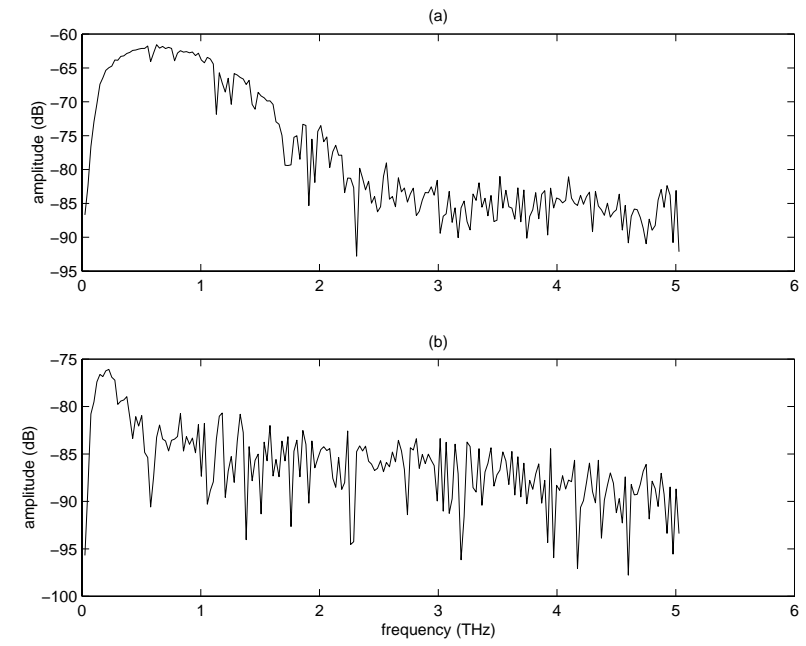

Figure 12. Frequency spectrum of the responses for two pixels on a piece of Spanish ham ('Jamon Serrano'). (a) shows a fatty area, while (b) shows a meaty area.
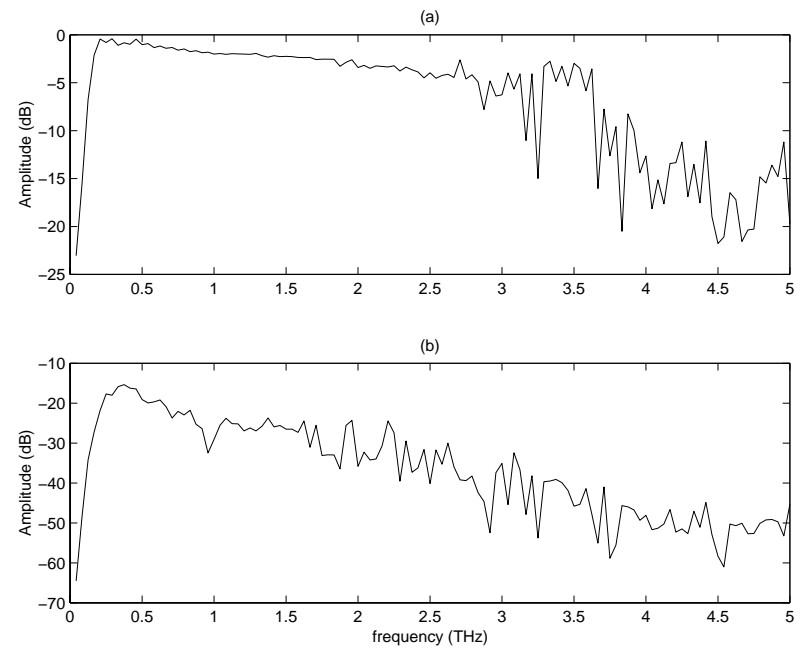

Figure 13. Frequency spectrum of ham T-ray pulses after Wiener deconvolution. (a) shows the fatty pixel, and (b) shows the meat pixel.

Wiener deconvolution has been illustrated previously for gas responses, ${ }^{6}$ the response of simple gas mixtures to terahertz radiation is relatively simple and can be accurately predicted, however the responses shown here are much more complicated as the response of biological samples is a combination of the molecular rotational absorption spectra and scattering for many different molecules. The data shown here is representative of the problem encountered when analysing human tissue. 
Figure 13 shows the results of Wiener deconvolution applied to the ham responses. It can be seen that the fat is relatively transparent to terahertz radiation so the deconvolved response is quite flat (up to $3 \mathrm{THz}$ ) whilst the meat has a complex terahertz response, deconvolution therefore makes the task of distinguishing between the two samples easier. It remains to develop classification metrics to allow the benefits of this method to be further analysed.

\section{COMPARISON OF TECHNIQUES}

(a)

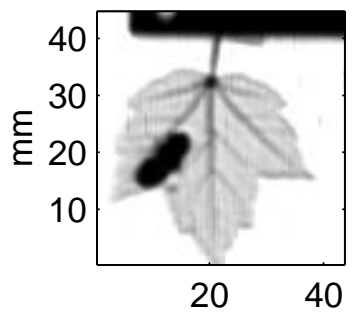

(b)

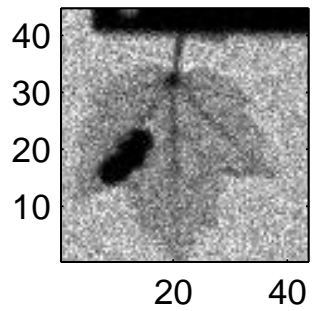

(c)

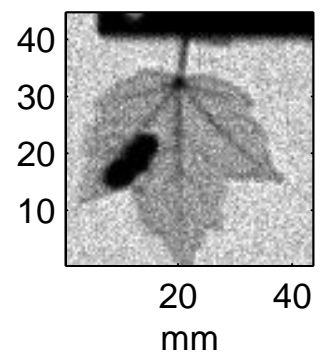

(d)

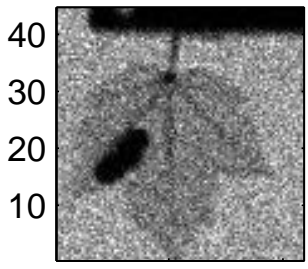

$20 \quad 40$ (e)

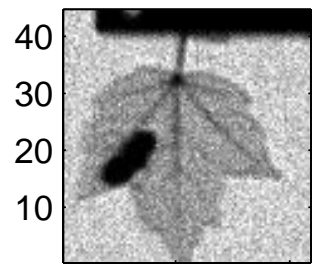

$20 \quad 40$

Figure 14. A comparison of T-ray images before and after various processing stages. (a) represents the original noiseless image (b) shows the same image after noise was added. (c) shows the noisy image after wavelet de-noising, (d) shows the noisy image after Wiener deconvolution and (e) shows the noisy image after wavelet de-noising followed by Wiener deconvolution.

To conclude this paper we give a visual comparison of the processing techniques discussed within this paper. We consider the original image shown in Figure 4 with noise added to each response such that the SNR was 3 dB. This is shown in Figure 14(b). The rest of the figure shows the result of wavelet de-noising, Wiener deconvolution and the combination of the two techniques on the image quality. The wavelet de-noising was performed using the procedure described in Section 5 using a Coiflet wavelet of order 4. The Wiener deconvolution was performed as described in Section 6 using the average free air pixel response as the system response. In all cases the image was produced by taking the Fourier transform of the processed T-ray pulses and plotting the magnitude of the Fourier coefficient corresponding to a frequency of $1 \mathrm{THz}$. This is an arbitrary scheme that produces good quality images. It can be seen that wavelet de-noising provides a marked improvement in image quality whilst deconvolution appears to offer little, however deconvolution promises greater benefits when classification algorithms are developed.

(a)

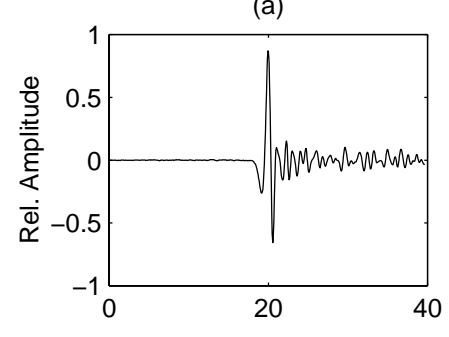

(b)

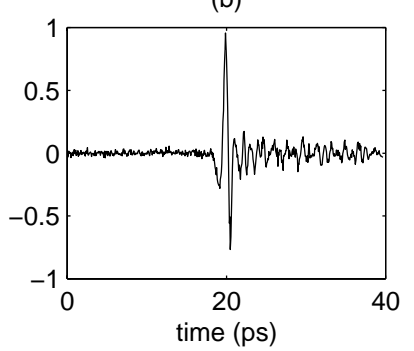

(c)

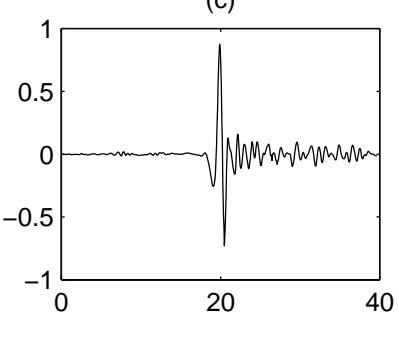

Figure 15. Illustration of wavelet de-noising of genuine noisy data. (a) shows the terahertz response of an Australian $\$ 100$ note measured with a LIA time constant of $100 \mathrm{~ms}$. (b) shows a noisy version of the same signal, measured with a LIA time constant of $1 \mathrm{~ms}$. (c) shows the result of wavelet de-noising applied to the signal in (b).

We also show the results of wavelet de-noising applied to the Australian $\$ 100$ note measurements shown earlier. Figure 15 shows the results of de-noising the signal measured with a small time constant (1ms) using a Coiflet order 4 wavelet. It can be seen that the noise is significantly reduced, the SNR was improved from $7 \mathrm{~dB}$ to $8.5 \mathrm{~dB}$. This technique therefore has the potential to allow effective systems to be constructed without the LIA, this would reduce 
the hardware complexity and, if the slow stepper motor that typically provides the optical delay is replaced by a scanning optical delay line, promises to dramatically increase the acquisition speed of the system. It also increases the power of the technique in measuring samples that have higher attenuation and correspondingly higher relative noise levels. This is of particular benefit to bio-medical applications where the attenuation is typically high.

\section{CONCLUSION}

This article has investigated the performance of signal processing techniques in analysing terahertz pulses obtained after transmission through biological samples. In particular the techniques of wavelet de-noising and Wiener deconvolution were analysed.

Soft wavelet de-noising was found to be well suited for this application, achieving up to 10dB improvement in SNR when applied to waveforms with initial SNRs of $3 \mathrm{~dB}$. An experimental investigation was performed to determine the ideal wavelet family and order for the de-noising application. The results of this experiment are not decisive but a number of useful conclusions were able to be drawn. Four wavelet families were compared and their performance, although similar could be ranked in order of success to yield Coiflet, Symlet, Daubechies and Meyer ordered by decreasing average resultant SNR. The order of wavelets within the families were also compared, where the order determines both the number of vanishing moments and the support width of the wavelet. The ideal order for each wavelet family was identified.

Wiener filtering was also applied to the problem of recovering the response of the sample from noisy data. The results of this analysis and the improvement in visual quality of T-ray images were discussed.

This is a dynamic field of research and much work remains. In particular signal processing strategies must be adapted to deal with $1 / \mathrm{f}$ noise originating from the ultrafast laser, and chirped, single shot terahertz responses. ${ }^{34,35}$ Many other signal processing techniques hold potential notably adaptive signal processing, and techniques adapted from speech recognition applications.

Ultimately these techniques, including wavelet de-noising and Wiener deconvolution must be evaluated against a classification criterion, and algorithms must be developed to accurately classify samples based on their terahertz responses.

\section{ACKNOWLEDGMENTS}

The authors would like to acknowledge the support of X.-C. Zhang's group at Rensselaer Polytechnic. In particular we wish to thank them for the provision of the oak leaf image data. The assistance of Qin Chen, RPI, in obtaining the Spanish ham measurements is greatly appreciated. We also appreciate communications with Doug Gray from the CRC for Sensor, Signal and Information Processing. Finally, we gratefully acknowledge fruitful discussions with Sam Mickan, Sheila Messer and Greg Harmer from Adelaide University.

\section{REFERENCES}

1. M. C. Nuss, "Chemistry is right for T-rays," IEEE Circuits and Devices 12(2), pp. 25-30, 1996.

2. S. Hadjiloucas, L. S. Haratzas, and J. W. Bowen, "Measurements of leaf water content using terahertz radiation," IEEE Transactions on Microwave Theory and Techniques 47(2), pp. 142-149, 1999.

3. M. van Exter, C. Fattinger, and D. Grischkowsky, "Terahertz time-domain spectroscopy of water vapour," Optics Letters 14(20), pp. 1128-1130, 1989.

4. D. Arnone, C. Ciesla, and M. Pepper, "Terahertz imaging comes into view," Physics World (4), pp. 35-40, 2000.

5. D. M. Mittleman, M. Gupta, R. Neelamani, R. G. Baraniuk, J. V. Rudd, and M. Koch, "Recent advances in terahertz imaging," Applied Physics B: Lasers and Optics 68(6), pp. 1085-1094, 1999.

6. D. M. Mittleman, R. H. Jacobsen, R. Neelamani, R. G. Baraniuk, and M. C. Nuss, "Gas sensing using terahertz time-domain spectroscopy," Applied Physics B: Lasers and Optics 67(3), pp. 379-390, 1998.

7. M. van Exter and D. Grischkowsky, "Characterization of an optoelectronic terahertz beam system," IEEE Transactions on Microwave Theory and Techniques 38(11), pp. 1684-1691, 1990.

8. D. Grischkowsky, S. Keiding, M. van Exter, and C. Fattinger, "Far-infrared time-domain spectroscopy with terahertz beams of dielectrics and semiconductors," Journal of the Optical Society of America B: Optical Physics 7(10), pp. 2006-2015, 1990 . 
9. R. H. Jacobsen, D. M. Mittleman, and M. C. Nuss, "Chemical recognition of gases and gas mixtures with terahertz waves," Optics Letters 21(24), pp. 2011-2013, 1996.

10. M. Knott, "See-through teeth," New Scientist 162(2192), p. 22, 1999.

11. P. Altmeyer, K. Hoffmann, and M. Stücker, eds., Skin Cancer and UV Radiation, Springer-Verlag, Berlin, 1997.

12. V. P. Wallace, D. C. Crawford, P. S. Mortimer, R. J. Ott, and J. C. Bamber, "Specrophotometric assessment of pigmented skin lesions: methods and feature selection for evaluation of diagnostic performance," Physics in Medicine and Biology 45(3), pp. 735-751, 2000.

13. J. B. Fishkin, O. Coquoz, E. R. Anderson, M. Brenner, and B. J. Tromberg, "Frequency-domain photon migration measurements of normal and malignant tissue optical properties in a human subject," Applied Optics 36(1), pp. 10-20, 1997.

14. M. Li, X.-C. Zhang, G. D. Sucha, and D. J. Harter, "Portable terahertz system and its applications," in Proceedings of SPIE, vol. 3616, pp. 126-135, 1999.

15. L. Duvillaret, F. Garet, and J.-L. Coutaz, "A reliable method for extraction of material parameters in terahertz time-domain spectroscopy," IEEE Journal of Selected Topics in Quantum Electronics 2(3), pp. 739-746, 1996.

16. L. Duvillaret, F. Garet, and J.-L. Coutaz, "Highly precise determination of optical constants and sample thickness in terahertz time-domain spectroscopy," Applied Optics 38(2), pp. 409-415, 1999.

17. Z. Jiang, M. Li, and X. C. Zhang, "Dielectric constant measurement of thin films by differential time-domain spectroscopy," Applied Physics Letters 76(22), pp. 3221-3223, 2000.

18. K. R. Castleman, Digital Image Processing, Prentice-Hall, Englandwood Cliffs, NJ, 1996.

19. S. Mickan, D. Abbott, J. Munch, X. C. Zhang, and T. van Doorn, "Analysis of system trade-offs for terahertz imaging," Microelectronics Journal 31, pp. 503-514, 2000.

20. K. P. Cheung and D. H. Auston, "A novel technique for measuring far-infrared absorption and dispersion," Infrared Physics 26(1), pp. 23-27, 1986.

21. D. M. Mittleman, R. H. Jacobson, and M. C. Nuss, "T-ray imaging," IEEE Journal of Selected Topics in Quantum Electronics 2(3), pp. 679-692, 1996.

22. H. A. Haus and A. Mecozzi, "Noise of mode-locked lasers," IEEE Journal of Quantum Electronics 29(3), pp. 983-996, 1993.

23. A. Poppe, L. Xu, F. Krausz, and C. Spielmann, "Noise characterisation of sub-10-fs Ti:sapphire oscillators," IEEE Journal of Selected Topics in Quantum Electronics 4(2), pp. 179-184, 1998.

24. L. Duvillaret, F. Garet, and J. L. Coutaz, "Influence of noise on the characterization of materials by terahertz time-domain spectroscopy," Journal of the Optical Society of America B: Optical Physics 17(3), pp. 452-461, 2000 .

25. S. Mallat, "A theory for multiresolution signal decomposition: the wavelet representation," IEEE Transactions on Pattern Analysis and Machine Intelligence 11(7), pp. 674-693, 1989.

26. D. L. Donoho, "Unconditional bases are optimal bases for data compression and for statistical estimation," Applied and Computational Harmonic Analysis 1(1), pp. 100-115, 1993.

27. D. L. Donoho, "De-noising by soft thresholding," IEEE Transactions on Information Theory 41(3), pp. 613-627, 1995.

28. C. K. Chui, An Introduction to Wavelets, vol. 1 of Wavelet Analysis and its Applications, Academic Press, San Diego, CA, U.S.A., 1992.

29. S. Mallat and Z. Zhang, "Matching pursuits with time-frequency dicitionaries," IEEE Transactions on Signal Processing 41(12), pp. 3397-3415, 1993.

30. S. Mallat, A wavelet tour of signal processing, Academic Press, San Diego, CA, U.S.A., 2nd ed., 1999.

31. I. Daubechies, Ten Lectures on Wavelets, CBMS-NSF regional conference series in applied mathematics, SIAM, Philadelphia, U.S.A., 1992.

32. R. K. Young, Wavelet Theory and Its Applications, Kluwer Academic, Norwell, MA, U.S.A., 1993.

33. N. Kalouptsidis, Signal Processing Systems Theory and Design, John Wiley \& Sons, Inc., New York, 1997.

34. Z. Jiang and X.-C. Zhang, "THz pulse measurement with a chirped optical beam," in Nonlinear Optics '98: Materials, Fundamentals and Applications Topical Meeting, pp. 297-299, IEEE, (Princeville, HI, U.S.A.), 1998.

35. Z. Jiang and X.-C. Zhang, "Single-shot spatiotemporal terahertz field imaging," Optics Letters 23(14), pp. 11141116, 1998. 